\title{
Pistillody mutant reveals key insights into stamen and pistil development in wheat (Triticum aestivum $\mathrm{L}$.)
}

\author{
Zaijun Yang, Zhengsong Peng*, Shuhong Wei, Mingli Liao, Yan Yu and Zeyan Jang
}

\begin{abstract}
Background: The pistillody mutant wheat (Triticum aestivum L.) plant HTS-1 exhibits homeotic transformation of stamens into pistils or pistil-like structures. Unlike common wheat varieties, HTS-1 produces three to six pistils per floret, potentially increasing the yield. Thus, HTS-1 is highly valuable in the study of floral development in wheat. In this study, we conducted RNA sequencing of the transcriptomes of the pistillody stamen (PS) and the pistil (P) from HTS-1 plants, and the stamen (S) from the non-pistillody control variety Chinese Spring TP to gain insights into pistil and stamen development in wheat.

Results: Approximately $40 \mathrm{~Gb}$ of processed reads were obtained from PS, P, and S. De novo assembly yielded 121,210 putative unigenes, with a mean length of 695 bp. Among these high-quality unigenes, 59,199 (48.84\%) had at least one significant match with an existing gene model. A total of 23,263 , and 553 differentially expressed genes were identified in PS vs. P, PS vs. S, and P vs. S, respectively, with differences in expression greater than five-fold. Among the differentially expressed genes, 206 were highly correlated with stamen and pistil development. These genes include WM27B, DL, YAB1, YABBY4, WM 5, CER 1, and WBLH1, which have been implicated in flower development. The expression patterns of 25 differentially expressed genes were confirmed through quantitative real-time reverse transcription PCR.

Conclusions: Analysis of this transcriptome resource enabled us to characterize gene expression profiles, examine differential gene expression, and produce a candidate gene list related to wheat stamen and pistil development. This work is significant for the development of genomic resources for wheat, and provides important insights into the molecular mechanisms of wheat stamen and pistil development.
\end{abstract}

Keywords: Pistillody, Pistil, Stamen, RNA sequencing, Wheat

\section{Background}

Wheat (Triticum aestivum L.) is a major staple food crop in several parts of the world, in terms of its cultivation area and use as a food source. Increasing yield to meet the increasing global demand for the crop is the main goal of wheat production. One way to improve the wheat yield potential is to increase the grain number per spike and unit area [1,2]. For this purpose, wheat scientists have considered a wide range of genetic variations in the morphological structure of wheat to obtain high grain numbers per spike. These morphological variations

\footnotetext{
*Correspondence: pzs8833@163.com

Key Laboratory of Southwest China Wildlife Resources Conservation, College of Life Science, China West Normal University, Nanchong 637009, Sichuan, China
}

include supernumerary spikelets, multi-spikelet [3], and multi-row spikes [4]. Peng [5] selected a three-pistil (TP) mutant with normal spike morphology that produced three pistils per floret. Consequently, a floret could develop into three seeds, thereby increasing the seed number per spike. Meanwhile, the novel pistillody mutant, HTS-1, was screened from Chinese Spring TP (CSTP), which is a near-isogenic line of the common wheat variety Chinese Spring with the Pis1 gene derived from the TP mutant [6]. HTS-1 plants exhibit a novel phenotype that transforms all or parts of the stamen into pistils or pistil-like structures. In recent years, the alloplasmic lines N26 [7] and (cr)-CSdt7BS [8] have been used to determine the genetic and molecular mechanisms of wheat pistillody [9-12]. Nuclear-cytoplasm 
interaction [8,12] causes pistillody in N26 and (cr)CSdt7BS. However, pistillody in HTS-1 is caused by the interaction of the recessive karyogenes hts1 and hts2 [6]. Therefore, HTS- 1 is genetically different from the previously reported lines (cr)-CSdt7BS and N26. Wheat florets are considered extremely stable and have a few reported mutants. Previous studies on floret mutants only provided a superficial understanding of floral organ identity determination in wheat plants. Consequently, HTS-1 is a significant genetic material to study the floral development of wheat; this line also has the potential to increase wheat yield.

Compared with studies on the functions of single or few genes during flower development [13,14], the underlying genetic determinants that control flower development have received relatively little attention in wheat. Moreover, the genes and their corresponding expression patterns related to pistil and stamen development have yet to be reported. Previous studies on expressed sequence tag sequences generated a large number of cDNA sequences for the wheat TriFLDB database (http://trifldb.psc.riken. jp/index.pl), which contains approximately 16,000 fulllength cDNAs [15]. Traditional sequencing methods have been used on randomly selected cDNA clones from various tissues; however, these methods obtained a low coverage of less-abundant or rare transcripts, which usually have vital functions. A novel approach to transcriptome profiling, called RNA sequencing (RNA-seq) has been developed recently, this method is based on nextgeneration sequencing (NGS) technologies [16,17]. RNAseq has been widely applied in plant biology, particularly in model species, such as Arabidopsis [18], and crop plants, such as rice [19], maize [20], and wheat [21].
In the present study, we used RNA-seq to investigate and compare the transcriptomes of pistillody stamen (PS) and the pistil (P) from HTS-1 plants, and of the stamen (S) from the non-pistillody control variety CSTP. The results of this study provide insights into $\mathrm{P}$ and $\mathrm{S}$ development in wheat.

\section{Results}

Comparison of the morphological structures of PS, P, and $\mathrm{S}$

Peng et al. [6] observed pistillody in HTS-1. HTS-1 is a CSTP sib-line that carries the Pis1 gene. However, HTS1 plants exhibit different florets; i.e., some HTS-1 stamens turn into pistils or a combination of stamens and pistils. As shown in Figure 1-a, the anther-like structure bears a tuft of 'stigma hair' at the right. A normal pistil and stamen are shown in Figure 1-b and 1-c. To compare the structures of PS, P, and S, each part was sectioned longitudinally and examined for histological modifications. The $\mathrm{P}$ showed well-developed ovules (Figure 1-e) and $\mathrm{S}$ contained normal pollen grains (Figure 1-f). PS (partially transformed stamen) contained ovule-like structures and had a pistil-like form; however, the ovules were underdeveloped and sometimes contained deformed pollen grains (Figure 1-d).

\section{Transcriptome sequencing and de novo assembly}

Three cDNA libraries (PS, P, and S; Figure 1) were constructed with the respective total RNA from PS, P, and S., The prepared libraries were sequenced on an Illumina Hiseq 2000 platform. A total of 134,561,846, 122,116,204, and 152,071,674 bp paired-end reads were obtained for PS, P, and S, respectively, which corresponded to a total

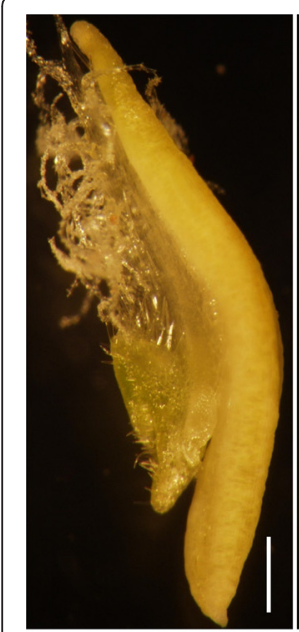

(a)

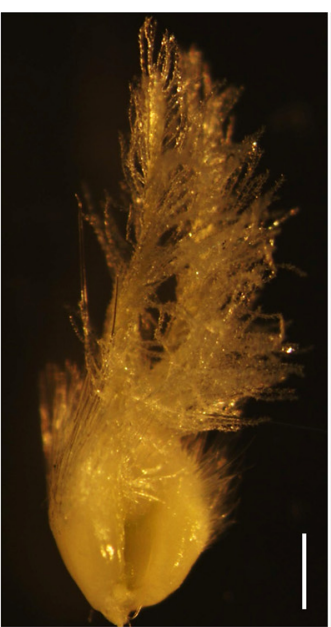

(b)

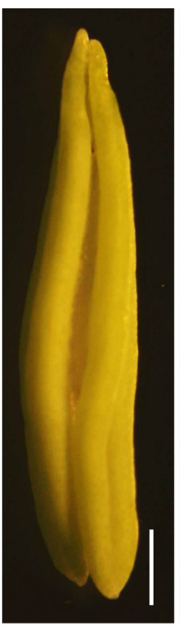

(c)

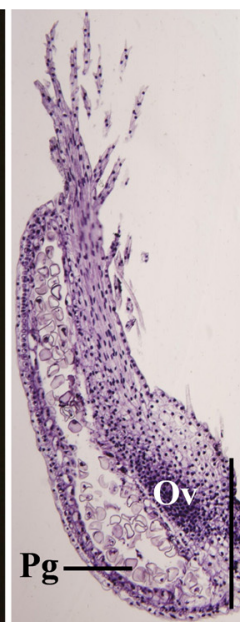

(d)

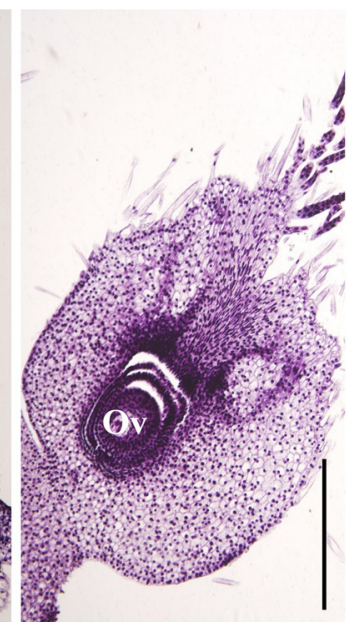

(e)

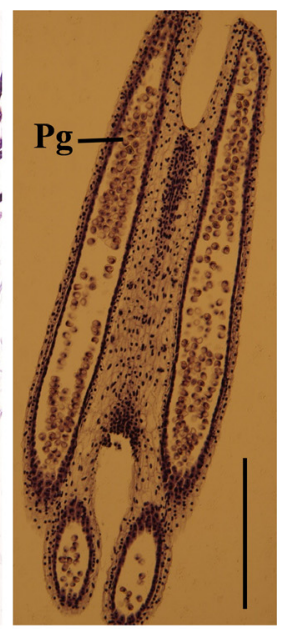

(f)

Figure 1 Comparison of morphological structure of pistillody stamen, pistil and stamen (a) pistilldoy stamen (b) normal pistil (c) normal stamen (d) longitudinal section of pistillody stamen (e) longitudinal section of normal pistil (f) longitudinal section of normal stamen. Ov: ovule, Pg: pollen grain. Bars $=1 \mathrm{~mm}$. 
size of $40.88 \mathrm{Gbp}$ after low-quality reads and adapter sequences were removed (Table 1). All paired-end reads were pooled together and assembled de novo using Trinity (v2012-10-05) [22]. Finally, we obtained 330,912 transcripts with lengths $\geq 200 \mathrm{bp}$. The mean length of these transcripts was $1,071 \mathrm{bp}$, and the maximum length was 20,603 bp (Table 1). The transcripts were assembled into 121,210 putative unigenes. The sequence information of all Illumina reads was deposited in the National Center for Biotechnology Information (NCBI) under the accession number SRP038912. The mean length of the putative unigenes was $695 \mathrm{bp}$. Among all the putative unigenes, 23,137 were longer than $1000 \mathrm{bp}$, which represented $19.1 \%$ of the total (Table 1). The size distribution of the assembly transcripts and unigenes is shown in Additional file 1: Figure S1. The 121,210 putative unigenes were aligned against the draft sequence of the bread wheat genome (IWGSP 1.21); 100,401 unigenes (82.2\%) could be mapped to the exon regions.

\section{Functional annotation of the unigenes}

The entire set of unigenes was annotated on the basis of their similarities with known or putative annotations in public databases, including GenBank NR, GenBank NT, $\mathrm{KO}$, SwissProt, PFAM, GO, and KOG (E values $\leq 1 \mathrm{e}-5$ for GenBank NR, GenBank NT, and SwissProt; E values $\leq 1 \mathrm{e}-3$ for KOG [23]). Among the 121,210 high-quality unique sequences, only 59,199 (48.84\%) had at least one significant match with an existing gene model in BLAST searches (Table 2).

Gene ontology (GO) was employed to identify the functional categories of the annotated unigenes and to classify the transcripts with known annotated proteins. 35,481 unigenes $(29.27 \%)$ had significant similarities to $\mathrm{Nr}$ and Pfam proteins and were assigned under GO terms. In several cases, multiple terms were assigned to

Table 1 Summary of RNA-seq and de novo assembly of $T$. aestivum unigenes

\begin{tabular}{ll}
\hline Number of clean reads & 408749724 \\
Number of $\geq 200$ bp transcripts & 330912 \\
Number of $\geq 2000$ bp transcripts & 48158 \\
Number of $\geq 1000$ bp transcripts & 131321 \\
Mean length of transcripts (bp) & 1071 \\
Max length of transcripts (bp) & 20603 \\
N50 length of transcripts (bp) & 1684 \\
Number of Unigenes & 121210 \\
Number of $\geq 2000$ bp Unigenes & 8553 \\
Number of $\geq 1000$ bp Unigenes & 23137 \\
Mean length of Unigenes (bp) & 695 \\
Max length of Unigenes (bp) & 20603 \\
N50 length of Unigenes (bp) & 1174 \\
\hline
\end{tabular}

the same transcript. The analysis produced 21,037 assignments to biological processes, 14,548 to cellular components, and 11,022 to molecular functions (Figure 2). Most of the biological process categories were related to metabolic processes (GO: 0008152, 23.23\%) and cellular processes (GO: 0009987, 23.68\%). Under the category of cellular components, $20.60 \%$ and $20.61 \%$ of the unigenes were located in cell parts (GO: 0044464) and cells (GO: 0005623), respectively. Among the molecular functions, the majority of the GO terms were grouped into either binding (GO: 0005488, 48.15\%) or catalytic activity (GO: 0008152, 37.55\%).

To further understand the biological functions and interactions of the gene products, a pathway-based analysis was conducted using the Kyoto Encyclopedia of Genes and Genomes (KEGG) pathway database. KEGG records the molecular interaction networks in cells with variants that are specific to particular organisms. Unigenes with annotated $\mathrm{Nr}$ and Pfam proteins were mapped to the KO database using KOBAS (KEGG Orthology-Based Annotation System, v2.0) [24]. The results showed that 11,848 unigenes could be assigned to 243 KEGG pathways for PS, P, and S transcriptomes (Additional file 2: Table S1). Most unigenes of the 243 KEGG pathways were ribosome pathways (324 genes). Glucosinolate biosynthesis, peptidoglycan biosynthesis, geraniol degradation, and polyketide sugar unit biosynthesis had the least number of unigenes (one gene).

\section{Differentially expressed genes (DEGs) among PS, P, and S}

The tag frequency differences that appeared in the PS, P, and $\mathrm{S}$ libraries were used to estimate the gene expression levels that corresponded to stamen and pistil development ( 0.3 reads per kilobase of transcript per million reads mapped was used as an expression threshold that was well above the background). The numbers of DEGs in PS vs. P, PS vs. S, and P vs. S were 95, 1,889 and 2,020 , respectively, for transcripts detected with $\mid \log 2$ fold change $\mid>2$ (Figure 3a). A total of 4,004 unigenes were identified as DEGs in at least two libraries. Among these shared DEGs, 1,626 common DEGs were found between PS vs. S and P vs. S, which may contribute to stamen and pistil development. The DEGs with $\mid \log 2$ fold change $\mid>5$ are shown in Figure 3b; at this level, PS vs. P, PS vs. S, and P vs. S had 23, 263, and 553 DEGs, respectively. Among the DEGs within this threshold, 206 DEGs were common in PS vs. S and P vs. S, including 83 upregulated genes and 123 downregulated genes (Additional file 3: Table S2). These 206 genes may have a high correlation with stamen and pistil development. PS vs. P had the least DEGs for $\mid \log 2$ fold change $\mid>2$ or $\mid \log 2$ fold change $\mid>5$, with only 95 and 23 DEGs, respectively. This trend suggested that the PS and P have similar transcript profiles. 
Table 2 Summary statistics of functional annotation for $T$. aestivum unigenes in public protein databases

\begin{tabular}{lll}
\hline & $\begin{array}{l}\text { No. of unigene } \\
\text { hits }\end{array}$ & Percentage (\%) \\
\hline Annotated in NR & 42865 & 35.36 \\
Annotated in NT & 39521 & 32.6 \\
Annotated in KO & 5491 & 4.53 \\
Annotated in SwissProt & 24130 & 19.9 \\
Annotated in PFAM & 30735 & 25.35 \\
Annotated in GO & 35481 & 29.27 \\
Annotated in KOG & 12087 & 9.97 \\
Annotated in all Databases & 2519 & 2.07 \\
Annotated in at least one Database & 59199 & 48.84 \\
Total Unigenes & 121210 & 100 \\
\hline
\end{tabular}

Functional annotation analysis of DEGs

The set of 206 common DEGs between PS $v s$. S and P vs. $\mathrm{S}$ was mapped in accordance with the GO biological process database to understand the functions of these DEGs; the gene expression profiles were compared with the whole transcriptome background to identify the genes involved in important biological processes. After GO Term Enrichment Analysis, 141 genes among the common DEGs were assigned to at least one term in the GO cellular component, molecular function, and biological process categories; these terms included 57 upregulated genes and 84 downregulated genes. Transcripts from the three categories were further classified into 25 functional groups, which provided an overview of the ontology of each transcriptome (Figure 4). Among the 25 functional groups, catalytic activity (33.5\%), metabolic process $(30.1 \%)$, and cellular process (29.1\%) were prominently represented. This result indicated that the three functional groups might be related to pistil and stamen development. At least seven unigenes with high

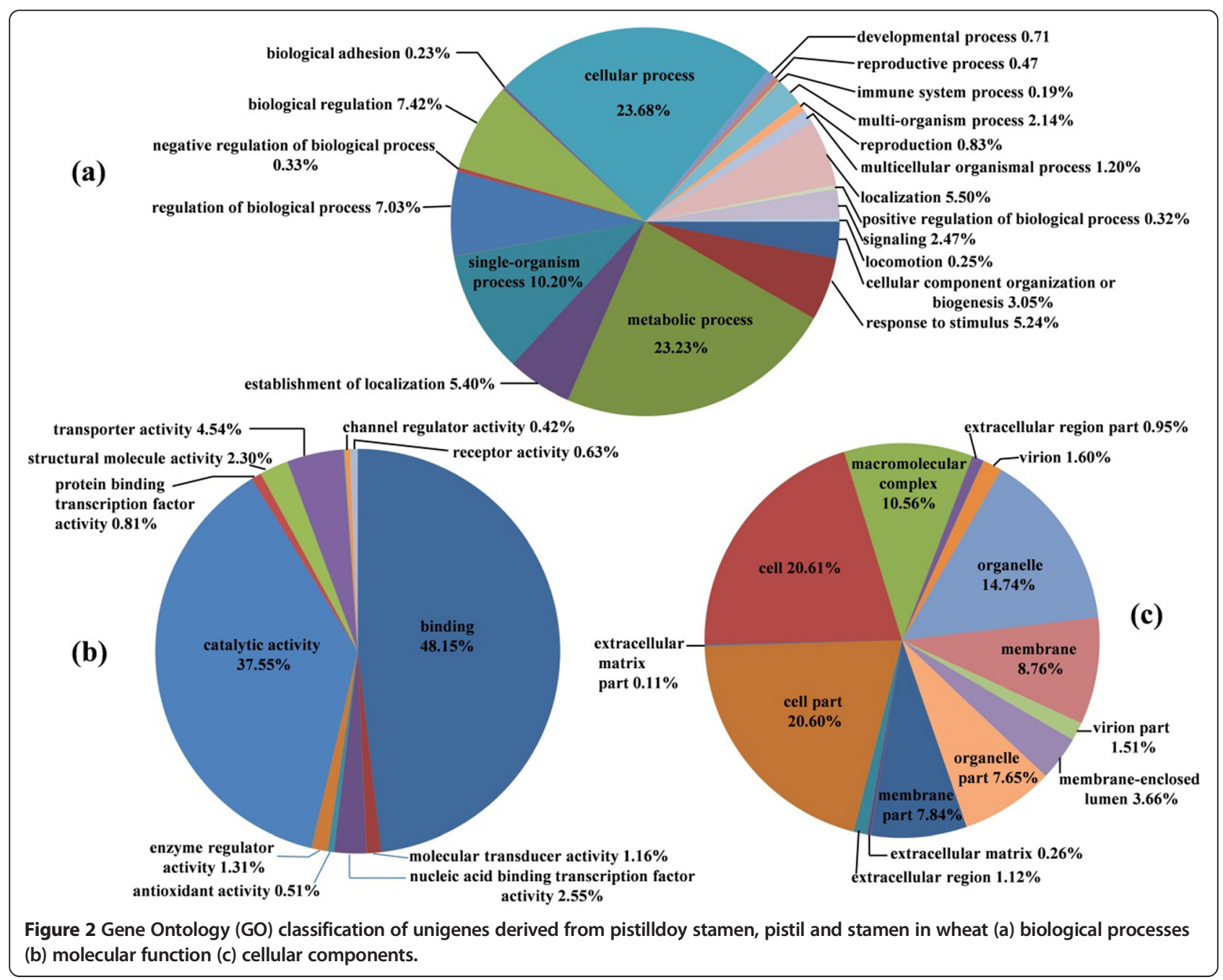




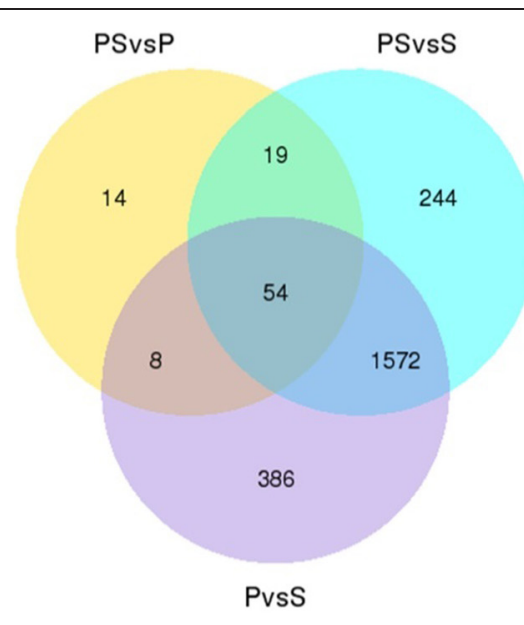

(a)

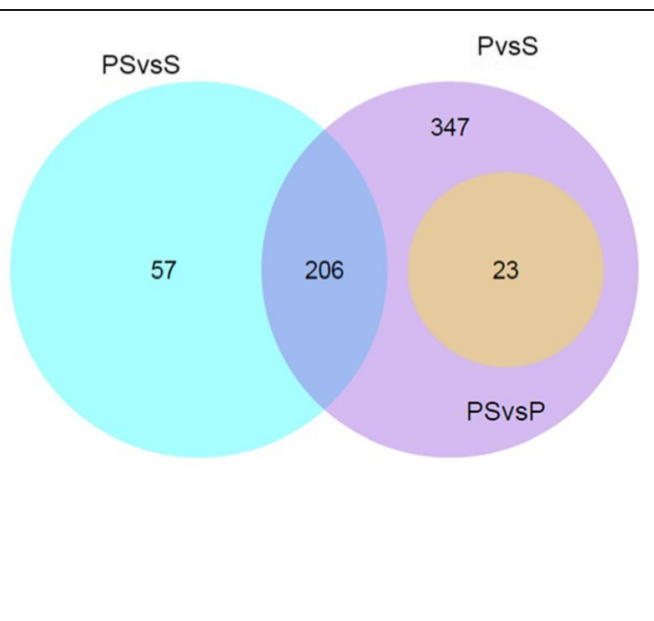

(b)

Figure 3 Venn diagram showing differentially expressed genes among pistillody stamen, pistil and stamen (a) transcripts detected with |log2 fold change $\mid>2$ (b) transcripts detected with |log2 fold change $>5$.

percentage identity to known flower development genes from wheat, barley, and Brachypodium distachyon (Table 3), were identified by comparing the DEGs found in this study with the NCBI databases.

\section{Quantitative real-time reverse transcription PCR (qRT-PCR) analysis}

qRT-PCR was performed on 25 unigenes, including 13 upregulated genes and 12 downregulated genes, to validate the results of expression profiling obtained from RNA-seq. Among the 25 unigenes, 18 unigenes were randomly selected from the 206 common DEGs, and the other seven unigenes were previously reported to influence flower development namely, MIKC-type MADSbox transcription factor $W M 27 B(T a A G-4)$, a $D L$ related gene, YAB1, YABBY 4, WM5, CER1, and WBLH1. We compared the results obtained from qRT-PCR with those generated from the RNA-seq analysis of the transcripts. The trends of expression were consistent for all transcripts in both analyses, with a correlation coefficient of $\mathrm{R}^{2}=0.9251$ (Figure 5). The expression profiles of WM27B, DL, YAB1, YABBY 4,WM5, CER1, and WBLH1 are listed in Table 4. The five genes, $W M 27 B$, $D L, Y A B 1, Y A B B Y$ 4, and $W M 5$, were indeed expressed at higher levels in PS and P compared with S (26 2339fold changes). CER1 and WBLH1 were downregulated genes in PS and S, and the transcript levels in S were 47 $\sim$ 1038-fold higher than in PS and P.

\section{Discussion}

Low-cost and high-throughput NGS technologies, particularly RNA-seq, have become useful, not only for de

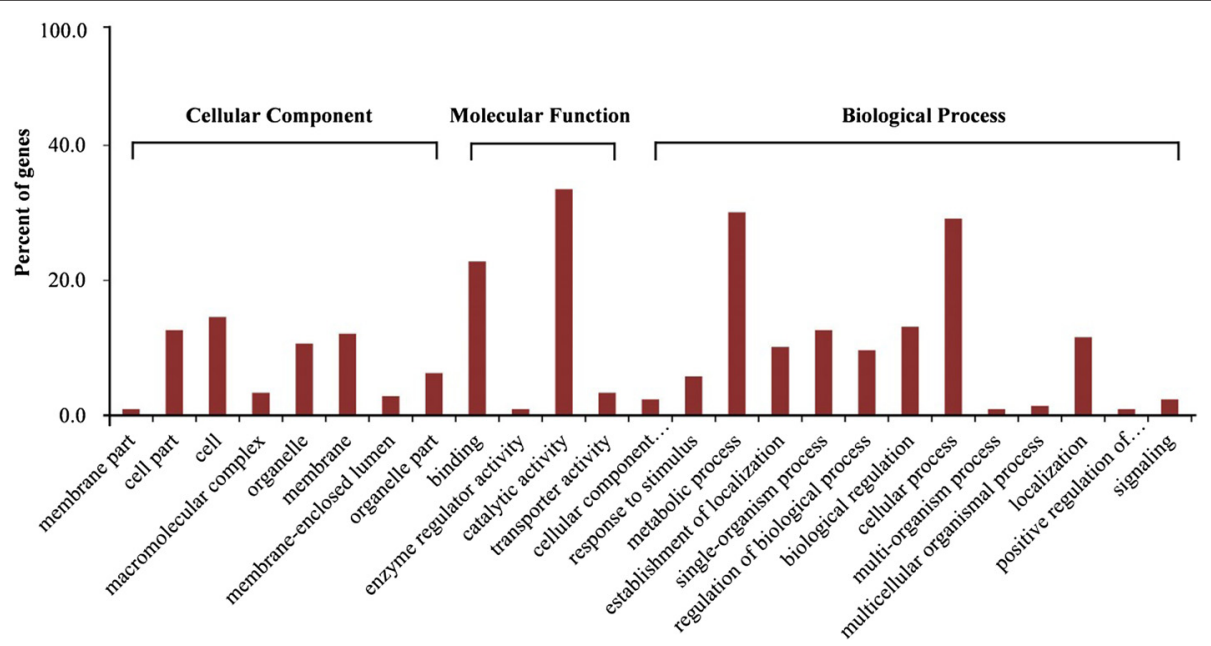

Figure 4 Gene Ontology (GO) annotations of common differentially expressed genes between PS vs S and P vs S. 
Table 3 List of previously reported genes to contribute to flower development

\begin{tabular}{|c|c|c|c|c|c|}
\hline Unigene ID & Size (bp) & $\begin{array}{l}\text { BLAST matching } \\
\text { accession No. }\end{array}$ & Gene description & $\begin{array}{l}\text { Sequence } \\
\text { identity (\%) }\end{array}$ & E value \\
\hline comp108229_c0 & 1098 & AM502895.1 & MIKC-type MADS-box transcription factor WM27B [Triticum aestivum] & 98 & 0 \\
\hline comp110066_c0 & 1385 & AB470269.1 & $D L$ related protein [Triticum aestivum] & 99 & 0 \\
\hline comp110106_c0 & 1718 & AY330228.1 & YABBY protein (YAB1) [Triticum aestivum] & 99 & 0 \\
\hline comp112313_c0 & 1680 & XM_003569922.1 & protein YABBY 4-like [Brachypodium distachyon] & 90 & 0 \\
\hline comp113007_c0 & 1600 & AY792974.1 & wheat meiosis 5 (WM5) [Triticum aestivum] & 97 & 0 \\
\hline comp112668_c1 & 1114 & DQ493457.1 & putative aldehyde decarbonylase enzyme CER1 [Hordeum vulgare] & 90 & 0 \\
\hline comp122918_c0 & 3622 & AB546642.1 & BEL1-type homeodomain protein WBLH1[Triticum aestivum] & 99 & 0 \\
\hline
\end{tabular}

novo genome assembly, molecular marker development, and genome diversity studies, but also to discover novel genes and to investigate gene expression profiles [16]. In model species, transcriptome profiling and gene expression quantification are generally performed by mapping reads from the NGS analysis to a reference genome sequence and then annotating the selected genes. The strategies for model species are not feasible in wheat because the reference genome sequence and gene annotation of wheat remain incomplete. However, an international project to achieve these goals is currently under way (International Wheat Genome Sequence Consortium, http://www.wheatgenome.org/). This project may take a considerable time because of the difficulties involved in sequencing the large (40 times larger than rice), highly repetitive, hexaploid genome of wheat. For this reason, biological analyses of wheat based on NGS data are challenging. The present study used NGS technology for wheat RNA-seq to characterize and compare the gene expression profiles among PS, P, and $\mathrm{S}$ to identify candidate genes related to pistil and stamen development.

In this study, Illumina sequencing from wheat PS, P, and $S$ produced 121,210 putative unigenes, of which only 59,199 unigenes (48.84\%) had at least one significant match with an existing gene models in BLAST searches (Table 2). This result may be attributed to the absence of the completely sequenced genome for wheat.

Common wheat $(2 n=6 \times=42)$ has a large genome (16 $\mathrm{Gb})$ and a high proportion of repetitive sequences (>80\%); therefore, it is difficult to de novo assemble the hexaploid wheat transcriptome. Oono et al. [21] verified the three de novo assembly tools (e.g., Trans-Abyss, Velvet-Oases, and Trinity) by comparing analyses from several programs using short-read sequence data obtained from wheat cultivar CS seedlings grown under reduced phosphorus. Their results indicated that Trinity produced the largest number of contigs and the longest

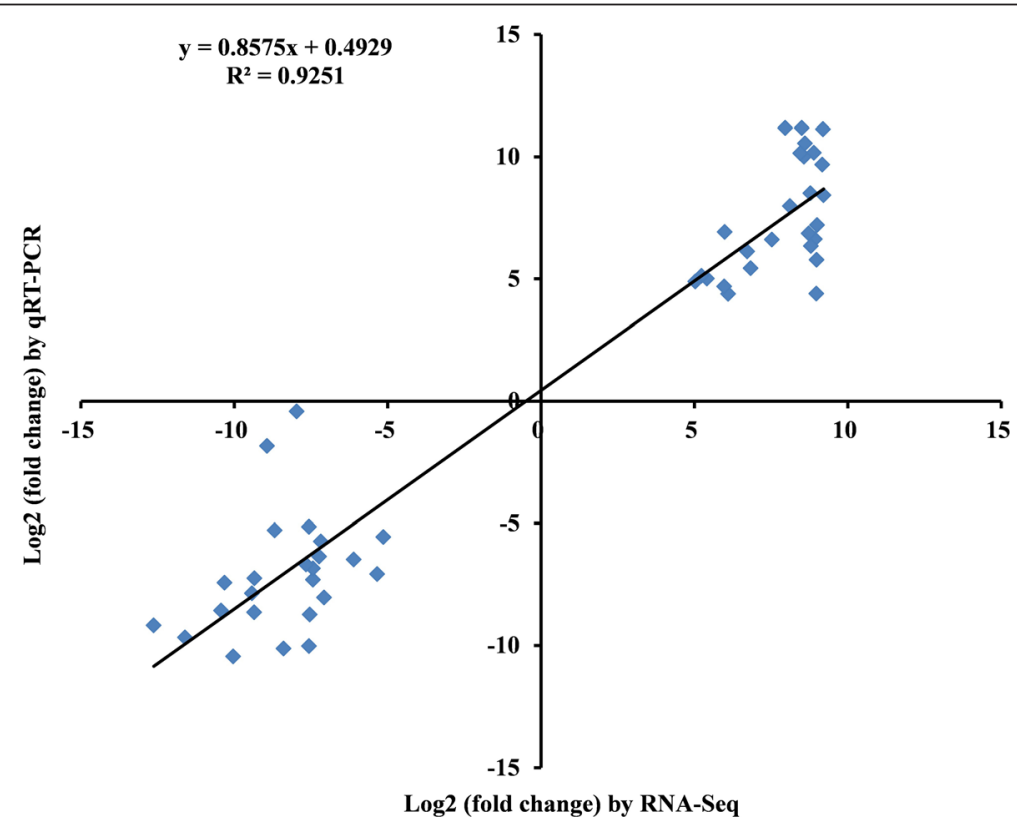

Figure 5 Comparison of expression levels measured by RNA-Seq and qRT-PCR for selected 25 transcripts from 206 common differentially expressed genes. 
Table 4 The expression profiling of previously reported genes to contribute to flower development

\begin{tabular}{|c|c|c|c|c|c|c|}
\hline \multirow[t]{2}{*}{ Unigene ID } & \multirow[t]{2}{*}{ Gene name } & \multicolumn{3}{|c|}{ Mean relative expression } & \multicolumn{2}{|c|}{ fold changes } \\
\hline & & PS & $\mathbf{P}$ & $\mathrm{S}$ & PS vs. S & P vs. S \\
\hline comp108229_c0 & WM27B & 0.64 & 1.33 & 0.03 & 21 & 44 \\
\hline comp110066_c0 & $\mathrm{DL}$ & 1.38 & 1.37 & 0.00059 & 2339 & 2322 \\
\hline comp110106_c0 & YAB1 & 1.53 & 1.23 & 0.013 & 118 & 95 \\
\hline comp112313_c0 & YABBY4 & 1.18 & 0.93 & 0.036 & 33 & 26 \\
\hline comp113007_c0 & WM5 & 1.78 & 1.52 & 0.051 & 35 & 30 \\
\hline comp112668_c0 & CER1 & 0.0013 & 0.0034 & 1.35 & $1 / 1038$ & $1 / 397$ \\
\hline comp122918_c0 & WBLH1 & 0.035 & 0.012 & 1.63 & $1 / 47$ & $1 / 136$ \\
\hline
\end{tabular}

unigenes. In this study, 121,210 unigenes, which were de novo assembled by Trinity, were aligned against the draft sequence of the bread wheat genome (IWGSP 1.21), and $82.2 \%$ unigenes could be mapped to the exonic regions. This result further justified the use of Trinity to de novo assemble the hexaploid wheat transcriptome.

Our analysis identified 206 DEGs, each with differences greater than five-fold, which were common in the comparisons of PS $v s . \mathrm{S}$ and $\mathrm{P} v s$. S. We deduced that these 206 genes may be highly correlated with stamen and pistil development because the PS and P have similar morphological structure and transcript profiles. The steady-state transcript levels for 25 genes were confirmed by qRT-PCR. Although the differences in gene expression based on qRT-PCR did not match the magnitude of those detected using RNA-seq, the upregulation and downregulation trends were similar. The lower expression levels detected by qRT-PCR could reflect the different sensitivities between the two technologies [25]. Illumina sequencing has been documented to be more sensitive for the estimation of gene expression, particularly for low-abundance transcripts, compared with microarrays and qRT-PCR [26]. Among the 206 genes, at least seven unigenes were previously reported to influence flower development: MIKC-type MADS-box transcription factor $W M 27 B(T a A G-4)$, a $D L$ related gene, $Y A B 1, Y A B B Y$ 4, WM5, CER1, and WBLH1.

The MADS-box transcription factor, WM27, belongs to the class $\mathrm{D}$ gene group and regulates ovule identity specification according to the "ABCDE" model in flower development [27]. In the present study, WM27 expression was upregulated in PS and P. This result is similar to the finding of Paolacci et al. [28] that TaAG-4 is highly expressed during late spike development, with weak expression in stamens, but high expression in pistils and caryopses. Therefore, the result demonstrated that WM27 may be involved in pistil development.

$Y A B 1$, the $D L$ related gene, and $Y A B B Y 4$ belong to the YABBY gene family; their upregulated expressions were detected in PS and S. The genes of YABBY family in Arabidopsis determine the abaxial cell fate of lateral organs [29]. In rice, YAB1 plays a major role in meristem development and causes extra stamens and carpels [30]. Thus, YAB1 may have an important function in wheat pistil and stamen development. Yamaguchi et al. [31] indicated that the $D L$ gene in rice interacts antagonistically with class B genes and controls floral meristem determinacy. Severe $D L$ loss-of-function mutations caused the complete homeotic transformation of carpels into rice stamens. No literature has indicated that the $Y A B B Y$ 4 gene is related to flower development. As a member of the YABBY gene family, YABBY 4 may have similar functions to other YABBY proteins. Moreover, the $Y A B B Y 4$ gene is upregulated in PS and P. Thus, YABBY 4 may contribute to pistil development.

Previous studies have shown that the wheat Meiosis 5 (WM5) gene is expressed in young flowers during meiosis, and encodes a novel protein that appears to be involved in meristem development, and flower and pollen formation [32]. According to Dong et al. [32], expression of the Meiosis 5 gene may be upregulated in stamens. However, Meiosis 5 showed upregulated expression in PS and P. Thus, the function of Meiosis 5 in pistil and stamen development remains unclear and needs further experimental verification.

The Arabidopsis CER1 gene encodes a novel protein involved in the conversion of long chain aldehydes to alkanes, which is a key step in wax biosynthesis. The cer1 mutants, which are conditionally male sterile, display glossy green stems and fruit [33]. CER1 was downregulated in PS and P or upregulated in S. This result indicated that CER1 is involved in stamen development. This result agrees with that of Aarts et al. [33].

The unigene comp122918_c0 is homologous to the wheat BEL1 gene WBLH1. Plant BEL1-like homeobox $(B L H)$ genes form a small gene family that functions in various developmental aspects, such as seed shattering in rice [34], or leaf shape establishment and ovule development in Arabidopsis [35,36]. Mizumoto et al. [37] isolated four $B L H$ genes in wheat, designated $W B L H 1$ to WBLH4. Expression analysis indicated that the WBLH1 gene is not related to ovule development. In the present 
study, the WBLH1 gene was downregulated in PS and P (upregulated in S). This result agrees with the finding of Mizumoto et al., [37]. Therefore, we speculated that WBLH1 has an important function in stamen development. In addition to the seven unigenes, the other 199 genes probably also contribute to stamen and pistil development. However, further research is required to determine the mechanisms by which these genes control wheat stamen and pistil development.

\section{Conclusions}

We sequenced and characterized the transcriptome of wheat PS, P from HTS-1 plants and S from the nonpistillody control CSTP. The use of this transcriptome resource enabled us to characterize gene expression profiles, examine differential expression profiles, and identify functional genes related to wheat stamen and pistil development. This work is significant for the development of genomic resources for wheat and provides important insights into the molecular mechanisms of wheat stamen and pistil development.

\section{Methods}

\section{Plant materials and RNA extraction}

HTS-1 is a novel common pistillody wheat mutant maintained in our laboratory. CSTP is a near-isogenic line of Chinese Spring, which is a common wheat variety that carries the Pis1 gene derived from the TP mutant [38]. HTS-1 was selected during the development process of CSTP [6]. Thus, CSTP and HTS-1 are siblines that show similar phenotypes, except for pistillody. CSTP and HTS-1 were cultivated in a field in the China West Normal University, Nanchong, China. Ten HTS-1 plants and ten CSTP plants were pooled as two samples, respectively. The PS (Figure 1a) and P (Figure 1b) in the HTS-1 pool, and the S (Figure 1c) in the CSTP pool at the heading stage were selected for RNA extraction. The PS, P, and S were independently collected twice, creating two biological replicates.

RNA was isolated from PS, P, and S using a modified cetyl trimethylammonium bromide-based protocol [39], with high salt concentrations and was further purified with the RNeasy Plant Mini Kit (Qiagen, Shanghai, China). An equal amount of total RNA was pooled from each plant for each wheat line. RNA quality and quantity were determined using a NanoDrop ND-2000 Spectrophotometer (Nanodrop Technologies, Wilmington, DE, USA) and were verified for degradation using a 2100 Bioanalyser RNA Nanochip (Agilent, Palo Alto, CA, USA).

\section{Morphological observation}

For the anatomical observations, the PS, P, and S in HTS-1 or CSTP at the heading stage were observed under a stereo microscope (Olympus, Tokyo, Japan). For light microscopy, the PS, P, and S were fixed in 50\% ethanol, $0.9 \mathrm{~mol} / \mathrm{L}$ glacial acetic acid and $3.7 \%$ formaldehyde at $4{ }^{\circ} \mathrm{C}$ for $15 \mathrm{~h}$. The specimens were stained with Alcian blue and dehydrated through a graded ethanol series, infiltrated with xylene, and then embedded in paraffin. A $12-\mu \mathrm{m}$ thick section was attached to the gelation-coated glass slides and observed under a light microscope (Olympus, Tokyo, Japan).

\section{cDNA library preparation and Illumina sequencing}

Three micrograms of RNA per sample was used as the input material for the RNA sample preparations. Sequencing libraries were generated using NEB $\mathrm{Next}^{\circ}$ Ultra $^{\text {TM }}$ Directional RNA Library Prep Kit for Illumina ${ }^{\circ}$ (San Diego, CA, USA) following the recommendations of the manufacturer. Four index codes were added to assign sequences for each sample. In brief, mRNA was purified from total RNA using poly-T oligo-attached magnetic beads. Fragmentation was performed using divalent cations under elevated temperature in the Illumina proprietary fragmentation buffer. First-strand cDNA was synthesized using random oligonucleotides and SuperScript II. Second-strand cDNA synthesis was subsequently performed using DNA polymerase I and $\mathrm{RNase} \mathrm{H}$. The remaining overhangs were converted into blunt ends via exonuclease/polymerase activities and the enzymes were removed. After adenylation of the 3 ' ends of DNA fragments, Illumina PE adapter oligonucleotides were ligated to prepare for hybridization. To select cDNA fragments of the preferred $200 \mathrm{bp}$ in length, the library fragments were purified using an AMPure XP system (Beckman Coulter, Beverly, CA, USA). DNA fragments with ligated adaptor molecules on both ends were selectively enriched using Illumina PCR Primer Cocktail in a 12-cycle PCR reaction. The products were purified (AMPure XP system) and quantified using the Agilent high-sensitivity DNA assay on the Agilent Bioanalyzer 2100 system (Agilent, Santa Clara, CA, USA).

The clustering of the index-coded samples was performed on a cBot Cluster Generation System using TruSeq PE Cluster Kit v3-cBot-HS (Illumina), in accordance with the manufacturer's instructions. After cluster generation, the library preparations were sequenced on an Illumina Hiseq 2000 platform and 100-bp paired-end reads were generated.

\section{De novo assembly}

Raw reads of the fastq format were first processed through in-house Perl scripts. In this step, clean reads were obtained by removing reads that contained an adapter and vector contamination (the reads were screened against the Univec database http://www.ncbi.nlm. nih.gov/VecScreen/UniVec.html), reads that contained 
poly-N sequences (number of poly-Ns greater than 10\%), and low-quality reads $(\mathrm{Q} 20, \mathrm{Q} 30$ and GC content were used for data filtering) from the raw data. The Trinity method [40] was used for the de novo assembly of wheat Illumina reads with the minimum kmer_cov set to 2 as the default, and all other parameters set to default. To avoid redundant transcripts, in-house Perl scripts were applied to extract the longest transcripts as unigenes. Unigenes generated with the assembly were used for downstream analysis. To evaluate the assembly strategies using Trinity, the unigenes were aligned to the draft sequence of the bread wheat genome (IWGSP 1.21, E value $<10^{-5}$ ).

\section{Functional annotation}

We annotated the unigenes based on a set of sequential BLAST searches, designed to find the most descriptive annotation for each sequence. The assembled unigenes were compared with sequences in the NCBI nonredundant $(\mathrm{Nr})$ protein and nucleotide $(\mathrm{Nt})$ databases (http://www.ncbi.nlm.nih.gov), the Protein family (Pram) database (http://en.wikipedia.org/wiki/Protein_family), the Cluster of Orthologous Group of proteins (KOG/COG) database (http://www.ncbi.nlm.nih.gov/COG), the SwissProt protein database (http://www.expasy.ch/sprot), the KEGG Ortholog (KO) database (http://www.genome.jp/ kegg/pathway.html), and the Gene Ontology (GO) database (http://www.geneontology.org/). Further functional enrichment analysis of DEGs was carried out using ToGO (Bioconductor package for R) (http://www.bioconductor. org/packages/release/bioc/html/topGO.html).

\section{Differential gene expression analysis}

Before differential gene expression analysis, the read counts were adjusted using an edgeR program package for each sequenced library through one scaling normalized factor. A differential gene expression analysis of two samples (PS vs. P, PS vs. S, and P vs. S) was performed using the DEGseq $R$ package. The $\mathrm{p}$ value was adjusted using a $q$ value [41]. Q Value $<0.005$ and $\mid \log 2$ fold change $\mid>2$ ware set as the threshold to judge the significance of gene expression difference. A large fold-change value ( $\mid \log 2$ fold change $\mid>5$ ) was also used to identify DEGs.

\section{Validation by qRT-PCR}

Twenty-five primer pairs (Additional file 4: Table S3) were designed to generate amplicons to validate the RNA-seq data. Aliquots of the total RNA extracted for sequencing were used for quantitative real-time PCR experiments in accordance with manufacturer's instructions (Qiagen, Shanghai, China). Real-time assays were performed with SYBR Green Dye (Takara, Dalian, China) using a Bio-Rad CFX96 real-time PCR platform (BioRad Laboratories, Hercules, CA, USA). All assays for a particular gene were performed three times synchronously under identical conditions, and RNA transcript fold changes were calculated through the $2^{-\Delta \Delta C t}$ method [42] with the wheat housekeeping genes Ubiq (DQ086482) and Actin (AB181911) as internal controls [13,43].

\section{Additional files}

Additional file 1: Figure S1. Distribution of assembly transcripts and unigenes lengths.

Additional file 2: Table S1. KEGG pathways represented in PS, P and S. Additional file 3: Table S2. The common differentially expressed genes between PS vs S and P vs S.

Additional file 4: Table S3. The primers designed for qRT-PCR.

\section{Competing interests}

The authors declare that they have no competing interests.

\section{Authors' contributions}

ZY and ZP conceived and designed the experiments; ZY and ZJ performed the tissue collection and RNA isolation; $S W, M L$, and $Y Y$ performed the data analysis; $Z Y$ performed the quantitative real-time PCR experiments; ZY wrote the manuscript drafts; ZP edited the manuscript; all authors read and approved the final manuscript.

\section{Acknowledgments}

This work was financially supported by the National Natural Science Foundation of China (Grant No. 31301319), and the key project of the Chinese Ministry of Education (Grant No. 211164). The funders had no role in the study design, data collection and analysis, publishing decision, or manuscript preparation.

Received: 13 September 2014 Accepted: 9 March 2015

Published online: 19 March 2015

\section{References}

1. Dencic S. Designing a wheat ideotype with increased sink capacity. Plant Breeding. 1994;112:311-7.

2. Frederic JR, Bauer PJ. Physiological and numerical components of wheat yield. In: Satorre EH, Slafer GA, editors. Wheat ecology and physiologyof yield determination (Chapter No. 3). New York: Food ProductsPress; 2000. p. 45-65.

3. Peng ZS, Yen C, Yang JL. Chromosomal location of genes for supernumerary spikelet in bread wheat. Euphytica. 1998;103:109-14.

4. Martinek P, Bednar J. Changes of spike morphology (multirowspike-MRS, long glumes-LG) in wheat (Triticum aestivum L.) and their importance for breeding. In: The proceedings of international conference 'genetic collections, isogenic and alloplasmiclines' Novosibirsk, Russia. 2001. p. 192-4.

5. Peng ZS. A new mutation in wheat producing three pistils in a floret. J Agron Crop Sci. 2003;189:270-2.

6. Peng ZS, Yang ZJ, Ouyang ZM, Yang $H$. Characterization of a novel pistillody mutant in common wheat. Aust J Crop Sci. 2013;7:159-64.

7. Murai K, Tsunewaki K. Photoperiod-sensitive cytoplasmic male sterility in wheat with Aegilops crassacytoplasm. Euphytica. 1993;67:41-8.

8. Murai K, Takumi S, Koga H, Ogihara Y. Pistillody, homeotictransformation of stamens into pistil-like structures, caused bynuclear-cytoplasm interaction in wheat. Plant J. 2002;29:169-82.

9. Meguro A, Takumi S, Ogihara Y, Murai K. WAG, a wheat AGAMOUS homolog, is associated with development of pistil-like stamens in alloplasmic wheats. Sex Plant Reprod. 2003;15:221-30.

10. Mizumoto K, Hatano H, Hirabayashi C, Murai K, Takumi S. Altered expression of wheat AINTEGUMENTA homolog, WANT-1, in pistil and pistil-like transformed stamen of an alloplasmic line with Aegilops crassacytoplasm. Dev Genes Evo. 2009;1219:175-87.

11. Saraike $T$, Shitsukawa $N$, Yamamoto $Y$, Hagita $H$, Iwasaki $Y$, Takumi $S$, et al. Identification of a protein kinase gene associated with pistillody, homeotic transformation of stamens into pistil-like structures, in alloplasmic wheat. Planta. 2007;227:211-21. 
12. Zhu Y, Saraike T, Yamamoto $Y$, Hagita H, Takumi S, Murai K. orf260cra, a Novel mitochondrial gene, is associated with the homeotic transformation of stamens into pistil-like structures (Pistillody) in Alloplasmic wheat. Plant Cell Physiol. 2008:49:1723-33.

13. Hama E, Takumi S, Ogihara Y, Murai K. Pistillody is caused by alterations to the class-B MADS-box gene expression pattern in alloplasmic wheats. Planta. 2004;218:712-20

14. Yang ZJ, Peng ZS, Wei SH, Yu Y. Cloning and characterization of endo- $\beta-1,4-$ glucanase genes in the common wheat line three pistils. Genet Mol Boil. 2013:36:400-7.

15. Mochida K, Yoshida T, Sakurai T, Ogihara Y, Shinozaki K. TriFLDB: a database of clustered full-length coding sequences from Triticeae with applications to comparative grass genomics. Plant Physiol. 2009;150:1135-46.

16. Varshney RK, Nayak SN, May GD, Jackson SA. Next generation sequencing technologies and their implications for crop genetics and breeding. Trends Biotechnol. 2009;27:522-30

17. Wang Z, Gerstein M, Snyder M. RNA-Seq: a revolutionary tool for transcriptomics. Nat Rev Genet. 2009;10:57-63.

18. Weber AP, Weber KL, Carr K, Wilkerson C, Ohlrogge JB. Sampling the Arabidopsis transcriptome with massively parallel pyrosequencing. Plant Physiol. 2007:144:32-42.

19. Lu T, Lu G, Fan D, Zhu C, Li W, Zhao Q, et al. Function annotation of the rice transcriptome at single-nucleotide resolution by RNA-seq. Genome Res. 2010;20:1238-49.

20. Li P, Ponnala L, Gandotra N, Wang L, Si Y, Tausta SL, et al. The developmental dynamics of the maize leaf transcriptome. Nat Genet. 2010;42:1060-7.

21. Oono Y, Kobayashi F, Kawahara Y, Yazawa T, Handa H, Itoh T, et al Characterisation of the wheat (Triticum aestivum L.) transcriptome by de novo assembly for the discovery of phosphate starvation-responsive genes: gene expression in Pi-stressed wheat. BMC Genomics. 2013;14:77-91.

22. Grabherr MG, Haas BJ, Yassour M, Levin JZ. Full-length transcriptome assembly from RNA-Seq data without a reference genome. Nat Bio. 2011;29:644-52.

23. Altschul SF, Madden TL, Schäffer AA, Zhang J, Zhang Z, Miller W, et al. Gapped BLAST and PSI-BLAST: a new generation of protein database search programs. Nucleic Acids Res. 1997;25:3389-402

24. Xie C, Mao XZ, Huang JJ, Ding Y, Wu JM, Dong S, et al. CKOBAS 2.0: a web server for annotation and identification of enriched pathways and diseases. Nucleic Acids Res. 2011;39:316-22

25. Wu J, Zhang Y, Zhang H, Huang H, Folta KM, Lu J. Whole genome wide expression profiles of Vitis amurensis grape responding to downy mildew by using Solexa sequencing technology. BMC Plant Biol. 2010;10:234-49.

26. AC't Hoen P, Ariyurek Y, Thygesen HH, Vreugdenhil E, Vossen RHAM, de Menezes RX, et al. Deep sequencing-based expression analysis shows major advances in robustness, resolution and inter-lab portability over five microarray platforms. Nucleic Acids Res. 2008;36:e141.

27. Pinyopich A, Ditta GS, Savidge B, Liljegren SJ, Baumann E, Wisman E, et al. Assessing the redundancy of MADS-box genes during carpel and ovule development. Nature. 2003;424:85-8.

28. Paolacci AR, Tanzarella OA, Porceddu E, Varotto S, Ciaffi M. Molecular and phylogenetic analysis of MADS-box genes of MIKC type and chromosome location of SEP-like genes in wheat (Triticum aestivum L.). Mol Genet Genomics. 2007;278:689-708.

29. Bowman JL. The YABBY gene family and abaxial cell fate. Curr Opin Plant Biol. 2000;3:17-22

30. Jang S, Hur J, Kim SJ, An G. Ectopic expression of OsYAB1 causes extra stamens and carpels in rice. Plant Mol Biol. 2004:56:133-43.

31. Yamaguchi T, Nagasawa N, Kawasaki S, Matsuoka M, Nagato Y, Hirano HY. The YABBY gene DROOPING LEAF regulates carpel specification and midrib development in Oryza sativa. Plant Cell. 2004;16:500-9.

32. Dong C, Thomas S, Becker D, Lörz H, Whitford R, Sutton T, et al. WM5: Isolation and characterisation of a gene expressed during early meiosis and shoot meristem development in wheat. Funct Plant Biol. 2005;32:249-58.

33. Aarts MGM, Keijzer CJ, Stiekema WJ, Pereira A. Molecular characterization of the CER1 gene of Arabidopsis involved in epicuticular wax biosynthesis and pollen fertility. Plant Cell. 1995;7:2115-27.

34. Konishi S, Izawa T, Lin SY, Ebana K, Fukuta Y, Sasaki T, et al. An SNP caused loss of seed shattering during rice domestication. Science. 2006;312:1392-6.

35. Kumar R, Kushakappa K, Godt D, Oidkowich MS, Pastorelli S, Hepworth SR, et al. The Arabidopsis BEL 1-LIKE HOMEODOMAIN protein SAW1 and SAW2 act redundantly to regulate KNOX expression spatially in leaf margins. Plant Cell. 2007;19:2719-35.

36. Reiser L, Modrusan Z, Margossian L, Samach A, Ohad N, Haughn GW, et al The BELL1 gene encodes a homeodomain protein involved in pattern formation in the Arabidopsis ovule primordium. Cell. 1995;83:735-42.

37. Mizumoto K, Hatano H, Hirabayashi C, Murai K, Takumi S. Characterization of wheat Bell1-type homeobox genes in floral organs of alloplasmic lines with Aegilops crassa cytoplasm. BMC Plant Biol. 2011;11:2-17.

38. Yang ZJ, Peng ZS, Zhou YH, Peng $\amalg$, Wei SH. Evaluation on the background of wheat near isogentic line for three pistils character by SRAP markers. J Nucl Agri Sci. 2012;26:22-7.

39. Chang SJ, Puryear J, Cairney J. A simple and efficient method for isolating RNA from pine trees. Plant Mol Biol Rep. 1993;11:113-116.

40. Grabherr MG, Haas BJ, Yassour M, Levin JZ, Thompson DA, Amit I, et al. Full-length transcriptome assembly from RNA-Seq data without a reference genome. Nature Biot. 2011;29:644-52.

41. Storey JD, Tibshirani R. Statistical significance for genome wide studies. Proc Natl Acad Sci. 2003;100:9440-5.

42. Livak KJ, Schmittgen TD. Analysis of relative gene expression data using real-time quantitative PCR and the $2^{-\Delta \Delta C t}$ method. Methods. 2001;25:402-8.

43. Yamada K, Saraike T, Shitsukawa N, Hirabayashi C, Takumi S, Murai K. Class D and $\mathrm{B}_{\text {sister }}$ MADS-box genes are associated with ectopic ovule formation in the pistil-like stamens of alloplasmic wheat (Triticum aestivum L.). Plant Mol Biol. 2009;71:1-14.

\section{Submit your next manuscript to BioMed Central and take full advantage of:}

- Convenient online submission

- Thorough peer review

- No space constraints or color figure charges

- Immediate publication on acceptance

- Inclusion in PubMed, CAS, Scopus and Google Scholar

- Research which is freely available for redistribution 